\title{
Reliability Sensitivity Analysis of Bearing-Rotor System Based on the Dynamic Coefficient
}

\author{
Qunchao Zhao ${ }^{1, a}$, Yimin Zhang ${ }^{1, b}$ \\ ${ }^{1}$ School of Mechanical Engineering and Automation, Northeastern University, Shenyang, Liaoning, \\ PR China 110819 \\ aqchzhao@mail.neu.edu.cn, bzhangymneu@sohu.com
}

Keywords: Reliability sensitivity, Sliding bearing, Dynamic characteristic, Rotor system instability

\begin{abstract}
According to components reliability sensitivity theory methods, looking upon the dynamic characteristics of hydrodynamic lubrication sliding bearing as the target for study, doing some reliability sensitivity analysis for the bearing-rotor system instability issues, numerical simulation results are given though analyzing. The outcome of the dynamic performance index is proposed based on the sliding bearing selection method for engineering practice to the selection of the radial journal bearing and provides a theoretical basis for overall design of shafting.
\end{abstract}

\section{Introduction}

The oil film instability is the key to the shaft system design in the radial journal bearing under hydrodynamic lubrication. It is often to get the instability speed of the shaft system by the theoretical analysis and calculated it before purchase or production in projects in order to consider in practical design to avoid or try overcoming. In existing studies, a lot of works are done on estimating and calculating the instability speed of the shaft system. On the basis of those above, it hopes to do some reliability sensitivity analysis on the index of the dynamic characteristics which affect the instability speed of the sliding bearing [1,2], and compare the result of those analysis under the difference initial parameters, further clear how the dynamic characteristics affect the shaft system instability and achieve a more rational bearing-rotor system.

\section{Angular instability of radial journal bearing under hydrodynamic lubrication}

The main principle of radial journal bearing under hydrodynamic lubrication is the so-called "oilwedge effect", which makes the shaft system in a certain speed appearing "nest move at half speed" [3]. The speed of shaft rotational that "nest moving at half speed" corresponds to is our concern here. Angular instability of radial journal bearing under hydrodynamic lubrication is as follow:

$$
\omega_{s}=\omega_{k 1}\left[P \pm \sqrt{P^{2}-\left(\frac{1}{B}\right)}\right]
$$

where, $\omega_{\mathrm{k} 1}$ is the first natural frequency of rotor system

$$
\begin{gathered}
P=\frac{m \omega_{k 1}}{2 A H} \quad(m \text { is the rotor mass }) \\
H=\frac{2 \eta L}{\psi^{3}}
\end{gathered}
$$

where $\eta$ is lubricant viscosity, $L$ is bearing width, $\psi$ is Relative bearing clearance.

$k_{i j}, c_{i j}(i=x, y ; j=x, y)$ are film stiffness and damping coefficients under dimensionless.

$$
A=\frac{k_{y x} c_{x y}+k_{x y} c_{y x}-\left(k_{x x} c_{y y}+k_{y y} c_{x x}\right)}{c_{y y}+c_{x x}}
$$




$$
B=\frac{k_{y x} k_{x y}-\left(k_{x x}+A\right)\left(k_{y y}+A\right)}{c_{x x} c_{y y}+c_{y x} c_{x y}}
$$

\section{Reliability analysis}

Calculation for reliability. Under stress-strength interference model [4], the definition of limit state function about film instability is as follow:

$$
\mathrm{g}(\mathbf{X})=\omega_{s}-\omega=\omega_{k 1}\left[P \pm \sqrt{P^{2}-\left(\frac{1}{B}\right)}\right]-\omega
$$

where, $\omega$ is the rotor system operating frequency.

When we talk about the dynamic coefficient of bearing-rotor system, those 8 coefficients are seems as random variable, then it is gotten:

$$
\mathbf{X}=\left[\begin{array}{llllllll}
k_{x x} & k_{x y} & k_{y x} & k_{y y} & c_{i x x} & c_{x y} & c_{y x} & c_{i y y}
\end{array}\right]^{\mathrm{T}}
$$

The indicator of stability is as follow:

$$
\beta=\frac{\mu_{\mathrm{g}}}{\sigma_{\mathrm{g}}}=\frac{E[\mathrm{~g}(\mathbf{X})]}{\sqrt{\operatorname{Var}[\mathrm{g}(\mathbf{X})]}}
$$

where, $\mu_{\mathrm{g}}$, and $\sigma_{\mathrm{g}}$ is the mean and standard deviation of the limit state function $\mathrm{g}(\mathbf{X})$. The following $\mu, \sigma$ signify the mean and standard deviation, too. The definition of the reliability is:

$$
R=\Phi(\beta)
$$

in the formula, $\Phi(\bullet)$ is the standard normal distribution function.

Calculation for the indicator of stability. According to the second moment reliability index technology:

$$
\begin{array}{r}
\mathrm{E}[g(\mathbf{X})] \approx g\left(\mathbf{X}^{0}\right)+\left.\sum_{i=1}^{n} \frac{\partial^{2} g}{\partial X_{i}^{2}}\right|_{\mathbf{X}=\mathbf{X}^{0}} \operatorname{Var}\left(X_{i}\right) \\
\left.\operatorname{Var}[g(\mathbf{X})] \approx\left[\frac{\partial g}{\partial \mathbf{X}} \otimes \frac{\partial g}{\partial \mathbf{X}}\right]\right|_{\mathbf{X}=\mathbf{X}^{0}} \operatorname{Cov}\left(X_{i}, X_{j}\right)
\end{array}
$$

The symbol " $\otimes$ " represents the Kronecker product, which is defined as

$$
(\mathbf{A})_{\mathrm{p} \times \mathrm{q}} \otimes(\mathbf{B})_{\mathrm{s} \times \mathrm{t}}=\left[\alpha_{\mathrm{ij}} \mathbf{B}\right]_{\mathrm{ps} \times \mathrm{qt}}
$$

Take formula (6) into (8) and (9), those are gotten:

$$
\begin{aligned}
& \mathrm{E}\left[g\left(\mathbf{X}^{\mathrm{T}}\right)\right] \approx g\left(k_{x x} \quad k_{x y} \quad k_{y x} \quad k_{y y} \quad c_{i x x} \quad c_{x y} \quad c_{y x} \quad c_{i y y}\right)+\frac{\partial^{2} g}{\partial k_{x x}^{2}} \sigma_{k_{x x}}^{2}+\frac{\partial^{2} g}{\partial k_{x x}^{2}} \sigma_{k_{x y}}^{2} \\
& +\frac{\partial^{2} g}{\partial k_{x x}^{2}} \sigma_{k_{y x}}^{2}+\frac{\partial^{2} g}{\partial k_{x x}^{2}} \sigma_{k_{y y}}^{2}+\frac{\partial^{2} g}{\partial c_{x x}^{2}} \sigma_{c_{x x}}^{2}+\frac{\partial^{2} g}{\partial c_{x y}^{2}} \sigma_{c_{x y}}^{2}+\frac{\partial^{2} g}{\partial k_{y x}^{2}} \sigma_{k_{y x}}^{2}+\frac{\partial^{2} g}{\partial k_{y y}^{2}} \sigma_{k_{y y}}^{2} \\
& \operatorname{Var}\left[g\left(\mathbf{X}^{\mathrm{T}}\right)\right] \approx\left(\frac{\partial g}{\partial k_{x x}}\right)^{2} \sigma_{k_{x x}}^{2}+\left(\frac{\partial g}{\partial k_{x y}}\right)^{2} \sigma_{k_{x y}}^{2}+\left(\frac{\partial g}{\partial k_{y x}}\right)^{2} \sigma_{k_{y x}}^{2}+\left(\frac{\partial g}{\partial k_{y y}}\right)^{2} \sigma_{k_{y y}}^{2} \\
& +\left(\frac{\partial g}{\partial c_{x x}}\right)^{2} \sigma_{c_{x x}}^{2}+\left(\frac{\partial g}{\partial c_{x y}}\right)^{2} \sigma_{c_{x y}}^{2}+\left(\frac{\partial g}{\partial c_{y x}}\right)^{2} \sigma_{c_{y x}}^{2}+\left(\frac{\partial g}{\partial c_{y y}}\right)^{2} \sigma_{c_{y y}}^{2}
\end{aligned}
$$

\section{Analysis for reliability sensitivity}

The reliability sensitivity with respect to the mean values of the system parameters are derived as follows [6]:

$$
\begin{aligned}
& \frac{\mathrm{d} R}{\mathrm{~d} \overline{\mathbf{X}}^{\mathrm{T}}}=\frac{\partial R}{\partial \beta} \frac{\partial \beta}{\partial \mu_{g}} \frac{\partial \mu_{g}}{\partial \overline{\mathbf{X}}^{\mathrm{T}}} \\
& \frac{\mathrm{d} R}{\mathrm{~d} \operatorname{Var}(\mathbf{X})}=\frac{\partial R}{\partial \beta} \frac{\partial \beta}{\partial \sigma_{g}} \frac{\partial \sigma_{g}}{\partial \operatorname{Var}(\mathbf{X})}
\end{aligned}
$$


where, $\frac{\partial R}{\partial \beta}=\varphi(\beta) ; \frac{\partial \beta}{\partial \mu_{g}}=\frac{1}{\sigma_{g}} ; \frac{\partial \mu_{g}}{\partial \mathbf{X}^{\mathrm{T}}}=\left[\begin{array}{llll}\frac{\partial \bar{g}}{\partial X_{1}} & \frac{\partial \bar{g}}{\partial X_{2}} & \cdots & \frac{\partial \bar{g}}{\partial X_{n}}\end{array}\right]$;

$$
\frac{\partial \beta}{\partial \sigma_{g}}=-\frac{\mu_{g}}{\sigma_{g}^{2}} ; \frac{\partial \sigma_{g}}{\partial \operatorname{Var}(\mathbf{X})}=\frac{1}{2 \sigma_{g}}\left[\frac{\partial \bar{g}}{\partial \mathbf{X}} \otimes \frac{\partial \bar{g}}{\partial \mathbf{X}}\right]
$$

in the formula, $\varphi($ )is the standard normal probability density function.

Take $\mathbf{X}=\left[\begin{array}{llllllll}k_{x x} & k_{x y} & k_{y x} & k_{y y} & c_{i x x} & c_{x y} & c_{y x} & c_{y y}\end{array}\right]^{\mathrm{T}}$ and formula (6) into the calculation for analysis of reliability sensitivity, the value of reliability sensitivity will be gotten.

\section{Numerical examples}

For a rotor system supported by the radial journal bearing under hydrodynamic lubrication, some expected values of the variables are shown as table 1 and table 2 . The variations of coefficients are defined as 0.05 consistently. Try to analyze the reliability sensitivity of the rotor system under film instability.

Table 1 Expected values of the variables

\begin{tabular}{|c|c|c|c|c|c|c|c|c|}
\hline Ref & $K$ & & $m$ & \multicolumn{2}{|c|}{$\eta$} & $L$ & \multicolumn{2}{|c|}{$\psi$} \\
\hline & \multicolumn{2}{|c|}{$\mathrm{N} / \mathrm{m}$} & $\mathrm{kg}$ & \multicolumn{2}{|c|}{$\mathrm{N} \cdot \mathrm{s} / \mathrm{m}$} & \multicolumn{2}{|l|}{$\mathrm{m}$} & \\
\hline & \multicolumn{2}{|c|}{$1.1 \times 10^{8}$} & 1820 & \multicolumn{2}{|c|}{0.008} & 0.04 & & \\
\hline & & Table & Expecte & e of & Iamic & ffficient & \\
\hline$\omega(\mathrm{r} / \mathrm{min})$ & $k_{\mathrm{xx}}$ & $k_{\mathrm{xy}}$ & $k_{\mathrm{yx}}$ & $k_{\mathrm{yy}}$ & $c_{\mathrm{xx}}$ & $c_{\mathrm{xy}}$ & $c_{y x}$ & $c_{y y}$ \\
\hline 1200 & 0.459 & -0.279 & 1.035 & 0.634 & 0.708 & 0.501 & 0.501 & 1.960 \\
\hline 1500 & 0.185 & -0.236 & 0.579 & 0.174 & 0.501 & 0.191 & 0.191 & 1.139 \\
\hline 1800 & 0.322 & -0.293 & 0.760 & 0.318 & 0.665 & 0.371 & 0.371 & 1.471 \\
\hline 2100 & 1.050 & -0.161 & 2.463 & 2.611 & 1.032 & 1.146 & 1.146 & 4.463 \\
\hline 2400 & 1.942 & 0.038 & 4.785 & 6.131 & 1.672 & 2.182 & 2.182 & 8.611 \\
\hline
\end{tabular}

We can know that the dynamic coefficients are changed with the mutative speeds from table 2 . After calculation by program, those under $2100 \mathrm{r} / \mathrm{min}$ speed are gotten:

$\beta=4.980381$

$R=0.9999996$

The reliability sensitivity about the dynamic coefficient under $2100 \mathrm{r} / \mathrm{min}$ speed is as follow:

$$
\frac{\mathrm{d} R}{\mathrm{~d} \overline{\mathbf{X}}^{\mathrm{T}}}=\left[\begin{array}{l}
\partial R / \partial k_{x x} \\
\partial R / \partial k_{x y} \\
\partial R / \partial k_{y x} \\
\partial R / \partial k_{y y} \\
\partial R / \partial c_{x x} \\
\partial R / \partial c_{x y} \\
\partial R / \partial c_{y x} \\
\partial R / \partial c_{y y}
\end{array}\right]^{\mathrm{T}}=\left[\begin{array}{c}
2.0417319 \mathrm{E}-04 \\
5.6300918 \mathrm{E}-04 \\
4.7632704 \mathrm{E}-05 \\
1.3485859 \mathrm{E}-04 \\
-4.1575986 \mathrm{E}-04 \\
5.1751042 \mathrm{E}-05 \\
5.1751042 \mathrm{E}-05 \\
-9.6138057 \mathrm{E}-05
\end{array}\right]^{\mathrm{T}}
$$

Table. 3. Value of the reliability sensitivity (RS) about the dynamic coefficient under different speeds.

\begin{tabular}{lrrrrc}
\hline$\omega(\mathrm{r} / \mathrm{min})$ & 1200 & 1500 & 1800 & 2100 & 2400 \\
\hline$\partial \mathrm{R} / \partial k_{\mathrm{xy}}$ & $3.8127010 \mathrm{E}-10$ & 3.190438 & $2.3703981 \mathrm{E}-02$ & $2.0417319 \mathrm{E}-04$ & $2.2085117 \mathrm{E}-02$ \\
$\partial \mathrm{R} / \partial k_{\mathrm{xy}}$ & $1.1526510 \mathrm{E}-09$ & 15.43700 & $9.0086639 \mathrm{E}-02$ & $5.6300918 \mathrm{E}-04$ & $6.2695444 \mathrm{E}-02$ \\
$\partial \mathrm{R} / \partial k_{\mathrm{yx}}$ & $2.1958171 \mathrm{E}-10$ & -5.966572 & $-3.0308673 \mathrm{E}-02$ & $4.7632704 \mathrm{E}-05$ & $1.1551321 \mathrm{E}-02$ \\
$\partial \mathrm{R} / \partial k_{\mathrm{yy}}$ & $3.7790002 \mathrm{E}-10$ & 4.251843 & $3.1095179 \mathrm{E}-02$ & $1.3485859 \mathrm{E}-04$ & $1.2385380 \mathrm{E}-02$ \\
$\partial \mathrm{R} / \partial c_{\mathrm{xx}}$ & $6.7379879 \mathrm{E}-10$ & -8.180470 & $-4.7190890 \mathrm{E}-02$ & $-4.1575986 \mathrm{E}-04$ & $-5.0600417 \mathrm{E}-02$ \\
$\partial \mathrm{R} / \partial c_{\mathrm{xy}}$ & $2.8665598 \mathrm{E}-11$ & -0.909246 & $-5.5187088 \mathrm{E}-03$ & $5.1751042 \mathrm{E}-05$ & $9.4618369 \mathrm{E}-03$ \\
$\partial \mathrm{R} / \partial c_{\mathrm{yx}}$ & $2.8665598 \mathrm{E}-11$ & -0.909246 & $-5.5187088 \mathrm{E}-03$ & $5.1751042 \mathrm{E}-05$ & $9.4618369 \mathrm{E}-03$ \\
$\partial \mathrm{R} / \partial c_{\mathrm{yy}}$ & $2.4339261 \mathrm{E}-10$ & -3.598258 & $-2.1333750 \mathrm{E}-02$ & $-9.6138057 \mathrm{E}-05$ & $-9.8250965 \mathrm{E}-03$ \\
\hline
\end{tabular}


The values of the RS about the dynamic coefficient under different speeds are shown as table 3. In order to compare horizontal and vertical, and give an intuitive way, fig. 1 and fig. 2 are given.

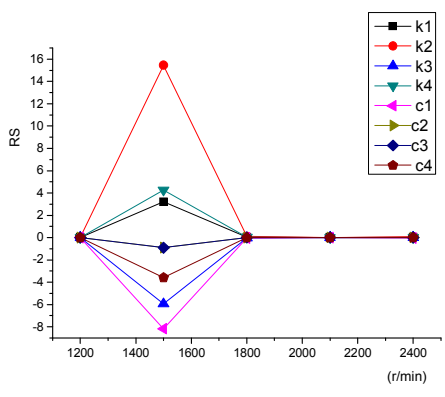

Fig. 1. RS under different working speeds

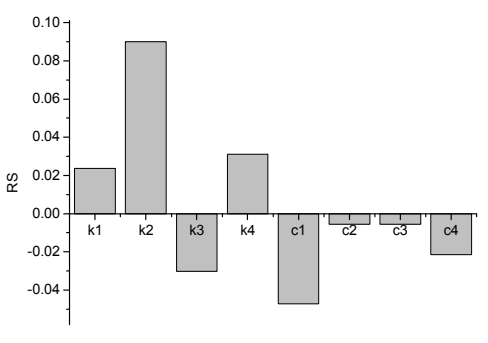

Fig. 2. RS under $1800 \mathrm{r} / \mathrm{min}$ working speed

Fig.1 describes the change of reliability sensitivity value about the dynamic characteristics under the different working speeds. Fig. 2 describes the reliability sensitivity value about the dynamic characteristics under the given working speed (1800 r/min). k1, k2, k3, k4, c1, c2, c3, c4 mean $\partial R / \partial k_{x x}, \partial R / \partial k_{x y}, \partial R / \partial k_{y x}, \partial R / \partial k_{y y}, \partial R / \partial c_{x x}, \partial R / \partial c_{x y}, \partial R / \partial c_{y x}, \partial R / \partial c_{y y}$. From fig.1, it can been seen obviously that dynamic characteristics' reliability sensitivity value changed significantly, when the working speed $(1500 \mathrm{r} / \mathrm{min})$ was close to this system's instability speed $(1526 \mathrm{r} / \mathrm{min})$. This phenomenon is consistent with engineering practice. As fig. 2 shown, $k_{x y}$ is the most significant factor which affects the whole system's reliability.

\section{Conclusions}

The approach of reliability sensitivity analysis of bearing-rotor system based on the dynamic coefficient is consistent with engineering reality. This analysis identified the dynamic coefficients $k_{x y}$ which is the greatest coefficient affecting on rotor bearing system stability. When designing and selecting bearings of rotor system, it should be tried to avoid setting the instability speed near the speed of work setting. If unavoidable, the effect of the change of dynamic coefficients on the system instability should been taken into account.

\section{Acknowledgements}

The work was supported by Natural Science Foundation of China (Grant no. 50875039) and the Changjiang Scholars and Innovative Research Team (no. IRT0816) by Ministry of Science and Technology of China.

\section{References}

[1] Y.M. Zhang, X.D. He, Q.L. Liu, B.C. Wen, Reliability-based Robust Optimization Design for Automobile Components Part 1 : Theory, Engineering Science, 2004 , 6 (3) : 75 79.

[2] Y.M. Zhang, Mechanical components reliability design, Beijing, 2000.

[3] L.X. Xu, Dynamic Design of High-speed rotating machinery shaft, Beijing, 1994.

[4] Y.X. Jiang, M.J. Zuo, Reliability model and Application, Beijing, 1999.

[5] S.W. Liu, Reliability Optimization Design of Mechanical Parts, Beijing, 1993.

[6] Y.M. Zhang, Q.L. Liu, Reliability - based design of automobile components, Proceedings of the Institution of Mechanical Engineers Part D , Journal of Automobile Engineering, 2002 , 216 (D6) : 455 471

[7] A.D. Belagunal, S. Zhang, Robust mechanical design through minimum sensitivity, Trans of the ASME, J. Mech Design, 1992,114 (6) : 213 217.

[8] H.M. Gomes, A.M. Awruch, Comparison of response surface and neural network with other methods for structural reliability analysis, Struct, Safety, 2004. 